\title{
X. On the resistance to the motion of small spherical bodies in elastic mediums
}

\section{Rev. J. Challis}

To cite this article: Rev. J. Challis (1832) X. On the resistance to the motion of small spherical bodies in elastic mediums, Philosophical Magazine Series 3, 1:1, 40-45, DOI: 10.1080/14786443208647817

To link to this article: http://dx.doi.org/10.1080/14786443208647817

册 Published online: 01 Jun 2009.

Submit your article to this journal $[\pi$

Џ Article views: 4

Q View related articles $₫$ 
fig. 24. In this way the elementary vortices in fig. 23. and 24. will explain the compound distributions of force in each individual case, as represented in the figures.

In fig. 16. and 17 , where the disc revolves on a centre, the excitation arising from the motion being in the direction $0 \mathrm{~m}$ on one side of the magnet, fig. 26, is counteracted by the opposite excitation on the other side of the line $o m$; for as on one side of the nagnet the motion would be advancing, and on the other side retiring, as in fig. 23. and 24. respectively, the forces arising therefrom would nearly, perhaps completely, destroy each other. It is possible, however, nay it is even probable, that all the systems of forces arising from the four rectilinear motions are in play when the disc is revolving on its axis; but the insignificancy of the two last contemplated forces, with regard to those which are due to the motions indicated by fig. 21. and 22, must necessarily render them exceedingly inefficient. If the force be electric, it is likely that the remote parts of the disc serve merely as conductors to that excited in the parts vicinal to the magnet.

The small curved arrows in fig. 19. and 20. indicate the distribution of the force in annular dises of copper or zinc, when rotated on an axis in the manner described for complete discs. The large exterior arrows indicate the direction of motion in each figure. The distribution in these annular discs is precisely the same, so far as the metal permits, as that in complete discs.

Fig. 27. is intended to show the position of the neutral line on the rectangular plate, when moved in the direction of the arrow between the magnetic poles. The arrow is a right line crossing the magnetic pole, and two inches in front of it. 'The small needtes are placed an inch from each other, and their positions, with regard to the arrow, show the inclination at each station, or the position in which the excited forces in the plate alone would place them.

X. On the Resistance to the Motion of small Spherical Bodies in elastic Mediums. By the Rev. J. Challis, Fellow of the Cambridge Philosophical Society*.

THE following observations have reference to the communication I made to the Philosophical Magazine and Annals for last March, and the mathematical reasoning therein contained, which being of a novel kind, requires to be con-

* Communicated by the Author. 
firmed in every possible way. I I shall here attempt to show that the results of that reasoning will serve to explain a phanomenon, which, as far as I know, has not yet received explanation.

These results were such as follow. If a disturbance be made in an elastic medium, in which the pressure is equal to the product of a constant $\left(a^{2}\right)$ by the density $(\rho)$, by means of a small sphere, the surface of which vibrates while its centre is fixed, and if $v=$ the velocity at the time $t$, at any point either at the disturbing surface or indefunitely near it, distant from the centre by $r$, then,

$$
\begin{aligned}
& v=\frac{\mathrm{F}^{\prime}(r-a t)}{r}-\frac{\mathrm{F}(r-a t)}{r^{2}} \\
& a \text { Nap. } \log \rho=\frac{\mathrm{F}^{\prime}(r-a t)}{r} .
\end{aligned}
$$

The former of these equations shows that $v$ is made up of two parts, $\frac{\mathrm{F}^{\prime}(r-a t)}{r}$, and $-\frac{\mathrm{F}(r-a t)}{r^{2}}$, distinguished from each other by the denominators $r$ and $r^{2}$. These denominators show that the velocity varies in passing at a given instant from the disturbing surface to a point indefinitely near, in a manner independent of the arbitrary function, and therefore of the disturbance also. We may perceive a natural reason for this, by considering that as the surface expands, the number of particles in contact with it is continually increasing, and to supply the increase the contiguous particles must have a motion towards the centre, independent of the motion they receive from the surface; and similarly when it contracts, a motion from the centre. Because $a \mathrm{Nap} . \log \rho$ is also equal to $\frac{\mathrm{F}^{\prime}(r-a t)}{r}$, it was inferred that this part of the velocity is propagated with the uniform velocity $a$. The other part, not being accompanied by change of density, is transmitted instantaneously, as if the fluid were incompressible.

We considered the case in which $\mathbf{F}(r-a t)=m \sin \frac{\pi}{\lambda}(r-a t)$, which applies to vibratory motion. Let us suppose for greater generality that $\mathrm{F}(r-a t)=m \times \sin \frac{\pi}{\lambda} \Phi(a t-r)$, and let $r$ be so small that terms involving higher powers than the first may be neglected. Then,

Third Series. Vol. 1. No. 1. July 1832. 


$$
\begin{aligned}
\mathbf{F}(r-a t) & =m \sin \frac{\pi}{\lambda}\left\{\Phi(a t)-\phi^{\prime}(a t) r\right\} \\
& =m\left\{\sin \frac{\pi \varphi(a t)}{\lambda}-\frac{\pi r \phi^{\prime}(a t)}{\lambda} \cos \frac{\pi \phi(a t)}{\lambda}\right\} \\
\mathrm{F}^{\prime}(r-a t) & =-\frac{\pi m \phi(a t)}{\lambda} \cos \frac{\pi \Phi(a t)}{\lambda}
\end{aligned}
$$$$
\text { and } v \text { or } \frac{\mathrm{F}^{\prime}(r-a t)}{r}-\frac{\mathrm{F}(r-a t)}{r^{2}}=\frac{m}{r^{2}} \sin \frac{\pi \Phi(a t)}{\lambda} \text {. }
$$

Now, if the motion of the disturbing surface, instead of being vibratory, be continually increasing or decreasing, $\lambda$ must be indefinitely great compared to $\pi \phi(a t)$ during the whole time of the motion: so that $\sin \frac{\pi \phi(a t)}{\lambda}=\frac{\pi \phi(a t)}{\lambda}$, and $\nu=\frac{\pi m \phi(a t)}{\lambda r^{2}}=\frac{u}{r^{2}} \phi(\alpha t)$, supposing that $\frac{\pi m}{\lambda}=\mu$. At the same time $\frac{F^{\prime}(r-a t)}{r}=-\frac{\pi m \phi^{\prime}(a t)}{\lambda r} \cos \frac{\pi}{\lambda} \varphi(a t)$ $=-\frac{u r}{r^{2}} \phi^{\prime}(a t)$, which on account of the factor $r \phi^{\prime}(a t)$ is very small compared to $v$. Hence in this case of disturbance, the part of the velocity accompanied by change of density is very small compared to the whole velocity, and therefore the change of density itself is very small.

Let, for example, $\varphi(\alpha t)$ be constant; then $v$ varies inversely as $r^{2}$, and $-\frac{u}{r^{2}} \cdot r \varphi^{\prime}(a t)=0$, as we should expect. Again, let $v$, which we may consider to be the arbitrary velocity given to the disturbing surface, be any function of the time, as $f(t)$, and let $r=r^{\prime}$ when $v=0$. Then $r=r^{\prime}+\int f(t) d t$, and $\mu \phi(a t)=v r^{2}=f(t)\left(r^{\prime}+\int f(t) d t\right)^{2}$. Hence it will be found that

$$
-\frac{u}{r} \phi^{\prime}(\dot{a} t)=-\frac{r f^{\prime}(t)}{a}-\frac{2 v^{2}}{a} .
$$

If $v$ be uniform, $f^{\prime}(t)=0$, and $-\frac{u}{r} \phi^{\prime}(a t)=-\frac{2 v^{8}}{a}$, which is a quantity of an order that has been already neglected. If $v=g t,-\frac{u}{r} \phi^{\prime}(a t)=-\frac{g r}{a}-\frac{2 v^{2}}{a}$, in 
which if $g$ represent the force of gravity, both the terms are negligible.

Conceive now a small spherical body to descend vertically in the air by the force of gravity. If it be supposed perfectly smooth, it can impress motion on the fluid only in directions perpendicular to its surface. Thus the motion impressed at each instant by the anterior half of the sphere is directed from a centre. If $v$ be the velocity of the sphere, $v \cos \theta$ is the velocity impressed in directions making an angle $\theta$ with the line of its motion. This case of disturbance is therefore similar to the last, in that the motion is from a centre; but differs in these respects, - the motion is not the same in all directions from the centre, and the centre is not fixed. But I have elsewhere given reasons for concluding (Cambridge Phil. Trans. vol. iil. part 3.) that the equations we have been using, and the results derived from them, apply at each instant to every elementary portion of fluid disturbed in any way, provided the condition of the tendency of the motion at each instant, to or from fixed or movesble centres, be fulfilled. If this be admitted, we may at once conclude that the descending sphere is subject to very little change of pressure on its anterior half; for if $\rho=$ the density of the fluid in contact with any point of it, we find that,

$$
a \text { Nap. } \log \rho=-\frac{r f^{\prime}(t)}{a}-\frac{2 v^{2}}{a},
$$

in which $f^{\prime}(t)=\frac{d v}{d t} \cos \theta$, a quantity not very different from $g \cos \theta$, since the resistance of the air is small. The same may be said of the posterior half; for it might be shown that the only difference between the disturbances produced by this half and the other, is that the motion is directed toroards a centre. Similar reasoning is applicable to any kind of increasing or decreasing motion. From all that precedes we draw this conclusion :-

When a small spherical body moves in a medium like air with a velocity small compared to the velocity of propagation in the medium, and in any manner except in rapid vibrations, the pressure on its surface is at every point very little different from the pressure of the medium at rest.

The phænomenon I propose to explain by this result is the spherical form of the drops of rain. That they are spherical is shown by the rainbow. Capillary attraction will account for their assuming in the first instance a spherical form; and from the preceding reasoning it follows that being very small, they do not suffer in passing through the air any inequality 


\section{Rev. J. Challis on the Resistance to the Motion, \&c.}

of pressure which will sensibly alter their shape. An inequality of pressure very much less than the weight of a drop would suffice to do this.

Hence also we may account for the success of the common method of making spherical shot, by letting them fall in a melted state from a great height, so as to become solid in their descent.

It appears from our reasoning that the resistance to a small spherical body descending in the air, is occasioned very little by the condensation of the air it encounters, but principally by its putting in motion and partly carrying with it a portion of the thid. Whatever be the law of resistance in regard to the velocity (which it seems difficult to ascertain), we may conceive of the nature of the resistance by supposing a variable mass $m f(v)$ to be always attached to the descending body $\mathrm{M}$, and to be unaffected by gravity; so that if $F=$ the effective accelerative force, $g \mathrm{M}=\mathrm{F}(\mathrm{M}+m f(v)) ; \mathrm{F}=\frac{g \mathrm{M}}{\mathrm{M}+m f(v)}$.

The foregoing inquiry will also assist us in ascertaining the nature of the resistance of the air to the motion of a pendulum-ball, suspended by a long slender thread. As before, the resistance is not sensibly due to any change of density of the air. The motion being slow and the vibrations of small extent, we may suppose, without chance of sensible error, that the velocity of a particle of the air in the same position relatively to the centre of the ball and the direction of its motion, has always the same ratio to the velocity of the ball. Hence if $\mathrm{M}$ be the mass of the ball, $\mu$ that of an equal volume of air, $m$ a certain constant, and $v$ the velocity of the ball, we have this equation of vis viva:

$$
\mathbf{M} v^{2}+m v^{2}=2 g(\mathbf{M}-\mu)(h-x),
$$

$h-x$ being the vertical descent of the centre of the ball. Hence the vertical accelerative force, or $-\frac{v d v}{d x}$, which is the only one that acts, is $g . \frac{M-\mu}{\mathbf{M}+m}$; and the tine of vibration is to the time in a vacuum, as $\sqrt{\frac{M+m}{M-\mu}}$ to 1 . These results have been obtained by M. Bessel. (Researches on the Length of the Seconds Pendulum: Berlin, 1828.)

The last application I propose to make of the preceding analysis, bears upon the nature of light. The undulatory hypothesis of light requires us to give a reason why the planets 
meet with no sensible resistance from the ethereal medium. Supposing the æther to be constituted like air, and the matter of the earth and planets to consist of very minute spherical atoms, (suppositions, which I have already advanced to explain some phænomena of light*,) it will follow that no part of the resistance, caused by the condensation of the medium, varies as the simple power of the velocity: also the term $-\frac{r f^{\prime}(t)}{a}$ must be quite insensible. With respect to that part of the motion of the æther which is unaccompanied by change of density, we may say that the velocity of any particle in the same position relatively to the centre of the planet, and to the direction of its motion, has always the same ratio to the velocity of the planet. 'The consequence of this would be, that the law of the force tending to the sun would not be changed, but its quantity would be diminished. This effect would be accounted for by taking the mass of the planet of less magnitude than it really is, and therefore probably cannot be easily detected by observations. Hence if any resistance be sensible by any change of the orbits or the periodic times, it will depend on the square of the velocity. Also it must be much less sensible in dense bodies like the planets, in which the particles that precede diminish the resistance on those that follow, than in such a rare substance as Encke's comet. This singular body has in fact, in the opinion of competent judges, determined the existence of a medium, or something equivalent, resisting according to the square of the velocity.

Papworth St. Everard, April 17, 1832.

XI. Nero Experiments relative to the Action of Magnetism on Electro-dynamic Spirals, and a Description of a nere Electromotive Battery. By Signor Salvatore Dal Negro; with Notes by Michael Faraday, Esq., F.R.S., M.R.I. Corr. Memb. Roy. Acad. Scien. of Paris, \& c. †

[Addressed to Dr. Ambrogio Fusinieri, Director of the Annali delle Scienze, \& c. \&c.]

Sir,

$\mathrm{O}$ repeating the experiments relative to the action of terrestrial magnetism on electro-dynamic spirals, an action which was first observed $\ddagger$ by the two illustrious Italian philo-

* See Phil. Mag. and Annals for March last, p. 161.

+ Communicated by Mr. Faraday.

* ['This is an crror. A long section is devoted to terrestrial magnetoelectric induction in my original researches (140 to 192) of the date of 